\title{
A Systematic Review and Meta-Analysis of En-Bloc vs Intralesional Resection for Giant Cell Tumor of Bone of the Distal Radius
} \author{
Forough Farrokhyar ${ }^{4}$ and Michelle Ghert ${ }^{*}, 1,2$ \\ ${ }^{I}$ McMaster University Department of Orthopaedic Surgery, Hamilton ON, Canada \\ ${ }^{2}$ Juravinski Cancer Centre, Hamilton ON, Canada \\ ${ }^{3}$ McGill University, Montreal QC, Canada \\ ${ }^{4}$ McMaster University Department of Surgery, Hamilton ON, Canada \\ ${ }^{5}$ The Saudi Ministry of Higher Education, Riyadh, Saudi Arabia
}

Theresa J.C. Pazionis ${ }^{1}$, Hussain Alradwan ${ }^{1,5}$, Benjamin M. Deheshi ${ }^{1,2}$, Robert Turcotte ${ }^{3}$,

\begin{abstract}
Introduction: Surgical management of Giant Cell Tumor of Bone of the distal radius (GCTDR) remains controversial due to risk of local recurrence (LR) offset by functional limitations which result from en-bloc resection. This study aims to determine the oncologic and functional outcomes of wide excision (WE) $v s$ intralesional curettage (IC) of GCTDR.

Methods: A complete search of the applicable literature was done. Included studies reported on patients from the same cohort who were surgically treated for GCTDR with WE or IC. Two reviewers independently assessed all papers. The primary outcome measure was LR.

Results: One-hundred-forty-one patients from six studies were included: 60 treated with WE, and 81 with IC. Five WE patients (8\%) suffered LR whereas 25 IC patients $(31 \%)$ did. The odds of LR were three times less in the WE group vs the IC group. MSTS1993 scores, where available, were on average 'good' with WE and 'excellent' with IC.

Conclusions: Within statistical limitations the data support an attempt, where feasible, at wrist joint preservation and superior function with IC. Intralesional curettage is reasonable when the functional benefit outweighs the risk of recurrence as is the case in many cases of GCT of the distal radius.
\end{abstract}

Keywords: Curettage, excision, giant cell tumor, meta-analysis, radius, surgery.

\section{INTRODUCTION}

Giant cell tumor of bone (GCT) is the most common benign aggressive primary bone tumor, accounting for approximately $5 \%$ of primary bone tumors in adults from ages 20 to 50 [1], with a slight predominance for females compared to males [1]. GCT may exhibit metastatic disease in less than 5\% of cases [2-5] most often to lung if observed. Even if metastatic, the course is generally indolent, although fatality has been reported [2].

The typical radiographic appearance of GCT is a lesion which is eccentrically located in the metaphyseal and epiphyseal regions of the long bones [1]. The hallmark appearance of GCT is an expansile lesion which is centrally radiolucent with formation of a thin neocortex at the border of the lesion [1]. Campanacci grade is the classification system of choice for giant cell tumors. Grade 1 tumors are confined within the cortex, grade 2 expand the cortex, and grade 3 perforate the cortex with resultant soft tissue

*Address correspondence to this author at the 699 Concession Street, Level 3 Surgical Oncology, Hamilton, ON, L8V5C2, Canada; Tel: 1-905-3899495, Ext. 64089; Fax: 905-575-6343; E-mail: michelle.ghert@ jcc.hhsc.ca extension [1]. Most GCTs are located in the knee region (distal femur and proximal tibia), and only $10 \%$ of cases occur in the distal radius [6]. The mainstay of treatment of GCT is surgery. Options include either en bloc resection and reconstruction, or intralesional curettage with a high speed burr, cryotherapy or phenolation, and cementation or bone grafting.

Giant cell tumor of the distal radius presents unique challenges. Some have suggested that these tumors more frequently recur than GCT in other locations [6] and functional outcomes are often adversely affected due to proximity to the carpus. Further, GCTs of the distal radius more frequently metastasize than those at other locations $[7$, 8]. The surgical management of GCT of the distal radius remains controversial due to the risk for local recurrence offset by the functional limitations which result from en-bloc resection. Appropriate surgical decision making dictates that for equivalent curative outcomes the least morbid procedure which maximizes functional outcomes should be performed. This discussion becomes increasingly important for benign aggressive tumors such as GCT which exhibit only limited metastatic potential [2-5]. Logically therefore it is difficult to justify not attempting an inital curettage and grafting 
procedure on Campanacci 1 and 2 GCT lesions of the distal radius. However, the question remains of defining a point where Campanacci 3 lesions treated with wide resection show improved functional outcomes and significantly decreased local recurrence than their counterparts treated with curettage and grafting. The objective of this study was to determine the oncologic and functional outcomes of enbloc $v s$ intralesional resection of the distal radius for GCT.

\section{MATERIALS AND METHODS}

\section{Design}

This study is a systematic review and meta-analysis. We evaluate and present the evidence regarding surgical treatment of giant cell tumor of the distal radius by either wide resection and reconstruction or intralesional curettage and cementation or grafting. Oncologic and functional outcome measures are evaluated.

\section{Literature Search}

Searches through MEDLINE, EMBASE, and the Cochrane Database were performed using the following search criteria: 'giant cell tumor', 'surgery' and 'distal radius'. For the EMBASE and Medline database searches, the same keywords (and variants) were used as both text words and Medical Search Headings (MeSH terms) and were combined by using Boolean operators as follows: ('exp Giant Cell Tumors' OR 'giant cell tumor.mp' OR 'giant-cell tumour.mp' OR 'giant cell tumor.mp' OR 'giant cell tumour.mp' OR 'GCT.mp') AND ('exp Forearm/ or forearm.mp' OR 'exp Radius/ or radius.mp' or 'exp Wrist/' or wrist.mp') AND ('surg.mp' OR 'excision.mp' OR 'resection.mp' OR 'curettage.mp or exp Currettage/' OR 'local control.mp'). The search was limited to papers published in English, and there were no restrictions on date of publication.

\section{Inclusion and Exclusion Criteria}

Studies were included if they reported on patients from the same cohort who were surgically treated for GCT of the distal radius with either en-bloc resection or with intralesional curettage. All Campanacci grades of GCT were included in the analysis. Ulnar lesions were excluded. Studies were excluded if the patient population were treated for tumors other than GCT and if all patients were treated with the same procedure (no comparative group). Comparative groups were defined as patients with equivalent disease states treated with a different procedure. Studies were also excluded if they reported less than 2 year followup or if they did not report recurrence as an outcome. Of note, locally recurrent lesions were included in our analysis.

\section{Outcome Measures}

The primary outcome evaluated was local recurrence. Secondary outcomes included pulmonary metastasis and functional outcomes where available. Data was extracted independently by two reviewers and discrepancies were reconciled by discussion.

\section{Assessment of Methodological Quality}

All eligible studies as determined by the inclusion and exclusion criteria were assessed for methodological quality by two independent assessors. The Newcastle-Ottawa Quality Assessment Scale (NOQAS) for cohort studies was used (http://www.ohri.ca/programs/clinical_epidemiology/o xford.htm) [9]. The inter-rater reliability and content validity have been established for this scale based on a critical review of the scale's ability to aid identification of articles suitable for a meta-analysis. This assessment scale evaluates papers on three key areas: selection of studies, comparability of the groups, and assessment of exposure (for case control) or outcome (for cohort studies). An independent statistician then calculated the level of agreement between the two independent assessors evaluations of the studies.

\section{Statistical Analysis}

The level of reviewer agreement of the NOQAS scores for study quality were assessed using calculation of the intraclass correlation coefficient. Further, the mean and standard deviation of NOQAS scores were calculated as reported in the results. Heterogeneity among studies was tested using the Cochrane $Q$ test with a $P$-value set at 0.1 for significance. The I-squared statistic, defined as the percentage of total variation across studies due to heterogeneity, was reported. We had planned a priori to use random effect model in the presence of heterogeneity and fixed effect model otherwise. Alpha was set at 0.05 for statistical significance. The pooled odds ratios and the $95 \%$ confidence intervals $(95 \% \mathrm{CI})$ for local recurrence and metastasis were calculated through the random-effects method by Review Manager version 5.1 (Cochrane Library.com).

\section{RESULTS}

\section{Literature Search}

Literature searches through MEDLINE, EMBASE, and the Cochrane Database yielded 2656 titles. The titles were

Table 1. Patient Demographics and Tumor Characteristics

\begin{tabular}{|c|c|c|c|c|c|c|c|c|c|c|c|c|}
\hline & \multicolumn{2}{|c|}{ Harness et al. } & \multicolumn{2}{|c|}{ Cheng et al. } & \multicolumn{2}{|c|}{ Steth et al. } & \multicolumn{2}{|c|}{ Panchwagh et al. } & \multicolumn{2}{|c|}{ VanderGriend $e t$ al. } & \multicolumn{2}{|c|}{ Kang et al. } \\
\hline Average age (years) & \multicolumn{2}{|c|}{$31+/-10$} & 30.83 & 41.3 & \multicolumn{2}{|c|}{34} & \multicolumn{2}{|c|}{36} & 29 & 32 & \multicolumn{2}{|c|}{38} \\
\hline M:F & \multicolumn{2}{|c|}{$20: 29$} & $1: 5$ & $2: 4$ & & 14 & \multicolumn{2}{|c|}{$13: 11$} & $3: 2$ & $2: 16$ & $5: 4$ & $5: 1$ \\
\hline Campanacci Grade $(1,2,3)$ & $(3,20,3)$ & $(0,12,7)$ & \multicolumn{2}{|c|}{ All grade 3} & \multicolumn{2}{|c|}{$(2,8,16)$} & $(1,8,3)$ & $(0,1,11)$ & & & \multicolumn{2}{|c|}{ All grade 3} \\
\hline
\end{tabular}

Legend: IC (Intralesional Curettage) ; WE (Wide Excision); M:F (Male to Female Ratio). 
screened for relevance to our topic and the search yielded 334 abstracts for review. The abstracts were screened based on the inclusion and exclusion criteria listed above, which yielded 20 papers which were reviewed in full. Fourteen papers were excluded due to no comparison group [10], tumors other than GCT being included [4], and ulnar lesions pooled in data tabulation [2]. Thus six papers were included in the analysis.

\section{Study Characteristics and Quality Assessment}

The results of our literature search yielded six retrospective cohort studies $[6,7,10,14,15,16]$, published from 1993 to 2010, which examined outcomes for both intralesional treatment and wide resection of GCT of the distal radius. Of the 141 patients included in the study, 60 $(42.6 \%)$ were treated with en- bloc excision and $81(57.4 \%)$ were treated with intralesional curettage. Thirty-seven $(26.2 \%)$ of the 141 patients in our study initially presented with locally recurrent tumor. Of these patients, $24(64.9 \%)$ were treated with en bloc resection and 13 (35.1\%) with intralesional curettage.

The indications to perform wide en bloc excision or intralesional curettage are not provided in the individual studies and therefore cannot be reported in this metaanalysis. Table $\mathbf{1}$ describes relevant patient and tumor characteristics. The six studies were assessed to be of moderate quality using the NOQAS scoring system. The mean and standard of scoring for reviewer 1 was 6.8 (SD +/.4) and for reviewer 2 was $7(\mathrm{SD}+/-0)$. Discrepancies in evaluation were discussed and reconciled as appropriate. The reliability could not be calculated due to the fact that the reviewers scores had almost zero variance

\section{Study Heterogeneity}

By using the local recurrence data for all studies, the Cochran's Q statistic was determined to be 0.06 with 3 degrees of freedom and a $\mathrm{P}$ value of 0.06 . There is significant heterogeneity between studies because the $\mathrm{P}$ value of 0.06 is less than alpha level of 0.10 as indicated for the test of heterogeneity. The calculated $\mathrm{I}^{2}$ for study heterogeneity was $0 \%$. However, the analysis was underpowered given the small number of events.

\section{Recurrence}

A total of 141 patients from six eligible studies were included in the analysis: 60 treated with en-bloc excision and 81 patients treated with intralesional curettage. Five patients
$(8 \%)$ in the en-bloc group suffered a local recurrence while 25 patients $(31 \%)$ in the intralesional group experienced a local recurrence. The odds ratio for local recurrence using the random effects models was 0.29 (95\% CI, 0.10-0.84, $\mathrm{P}=0.02)$ in favor of en-bloc excision for local control (Fig. 1).

\section{Metastasis}

In all but one of the studies [7], metastases did not occur within the follow up period. In this study, one patient from the intralesional group and one from the wide excision group each developed pulmonary metastatic disease and subsequently underwent thoracotomy [7]. Due to the low number of events, the pooled odds ratio could not be calculated.

\section{Functional Outcomes}

Functional outcomes as reported in the studies are summarized in Table 2 . Not all studies cited functional outcomes, however where stated, intralesional curettage was found to be equivalent or preferable to wide excision in terms of functional outcome. In the three studies reporting MSTS scores, the intralesional curettage group had overall higher MSTS scores than those of the wide excision group. Kang et al. [10] compared 'Disability of the Arm, Shoulder and Hand' (DASH) scores between the two groups, with no statistically significant difference. Visual Analogue Scale (VAS) for pain and percent grip strength, where reported, were however significantly improved in the intralesional curettage groups. Observationally this implies improved functional outcomes for intralesional curettage but there is significant heterogeneity in outcome reporting which limits our interpretation of these results.

\section{DISCUSSION}

Giant cell tumor of the distal radius is a unique entity. Although classified as benign aggressive, it does carry a relatively higher rate of recurrence than its counterparts in other long bones [11], and the anatomically sensitive area of the wrist joint is difficult to manage with a wide resection without morbidity. Intralesional curettage, with the possible use of adjuvants such as phenol or alcohol, and filling the cavity with either bone graft or cement, is a desirable treatment option.

The data examined in our meta-analysis show $8 \%$ of patients in the en-bloc group had a local recurrence while $31 \%$ in the intralesional group suffered a local recurrence.

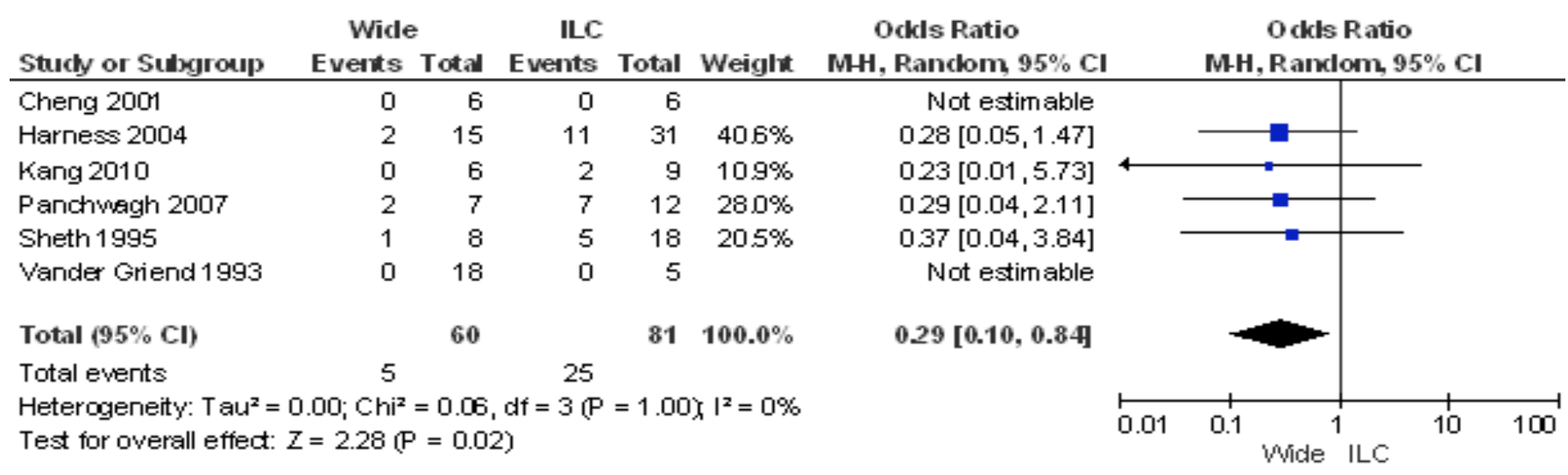

Fig. (1). Forest Plot for Local Recurrence. 
Table 2. Comparative Functional Outcomes

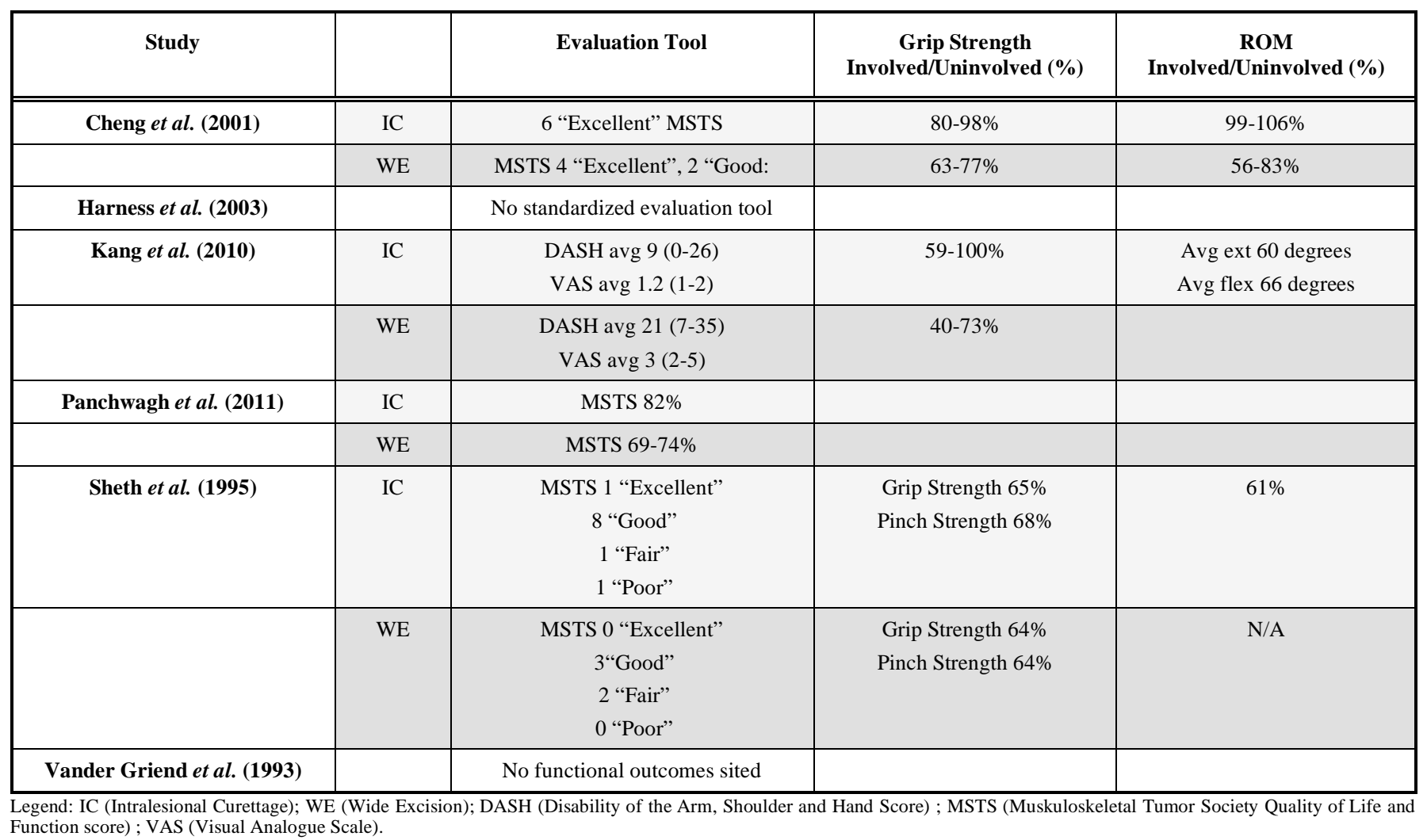

These statistics may imply that wide excision leads to improved reduction of tumor cell burden vis a vis intralesional curettage. We observed that en bloc excision was more frequently performed for Grade $3 \mathrm{GCT}$, which have more extensive tumor burden and cortical destruction. It is interesting to note that despite this, fewer recurrences are observed in this group. A likely explanation is that resection of the metaphysis results in more definite removal of all GCT cells thus decreasing the risk of recurrence, but one must also be aware that significant study heterogeneity may limit validity of results.

Conversely, upon examination of the functional data, we consistently observe improved functional outcomes as evaluated by MSTS scores or other functional measures, and a statistically significant difference in the VAS pain scores with improved scores in the intralesional group discussed in the Kang et al. [10] study. Therefore, although this study confirms a higher risk for local recurrence with intralesional curettage compared to en bloc excision, it also suggests suboptimal functional outcomes following more aggressive surgical resections. Interestingly, other studies have also found that intralesional resection in GCT results in fewer complications and better functional results then en bloc resection for GCT in other skeletal sites. [12]. However, due to the lack of consistency in reporting functional outcomes, the functional data cannot be pooled and these conclusions are somewhat speculative. The variability in analysis and data collected limits the validity of any generalized conclusions about functional outcome. Our current study is not powered to draw conclusions about functional outcome and we can only offer scientifically based opinion. However, an interesting area for further research would be to further focus on optimizing functional outcomes for lesions with equivalent campanacci grade and presumed local recurrence risk.

The data presented in our meta-analysis is echoed in other recently published reviews [13]. Liu et al. outline data presented in six articles reporting on 80 curettage cases and 59 en bloc excision cases. They found that patients undergoing intralesional curettage had a higher risk of recurrence especially in campanacci 3 tumors, but also fewer complications than the en bloc resection group. Complications reported included nonunion at the graft-radius junction, fracture of graft, skin necrosis, death, fragmentation with carpal collapse, and subluxation or arthrosis which impaired joint function.

Locally recurrent lesions were included in our analysis as there is evidence to show that recurrent lesions should actually be treated as primary lesions given they do not differ histologically [14], and recurrence is more than likely due to incomplete removal of tumor burden with initial treatment. We do observe proportionately more patients presenting with locally recurrent lesions being treated with WEE $(64.9 \%)$ compared to the entire sample (42.6\%). No definite conclusions can be drawn from this observation due to inconsistency in reported Campanacci grades and lack of information concerning these patient's original presentation and surgeries. It must be recognized that pooling locally recurrent and primary lesions introduces a potentially important source of bias into our study especially for those locally recurrent lesions included in the intralesional curettage group. Of note, the choice to perform curettage and grafting $v s$ a wide resection of recurrent lesions depends mostly on the extent of bone and soft tissue involvement. A 
further issue for discussion is the probability of selection bias for lesions undergoing intralesional curettage. Surgeons were more likely to have chosen this procedure over en bloc excision if the radiographic extent of the tumor suggested containment of the defect and therefore a lower chance of recurrence $[10,15]$. Therefore it is likely that patients who underwent intralesional curettage presented with radiographically less aggressive tumors and were therefore less likely to suffer a local recurrence with this treatment $[14,15])$. However, there is likely to be surgeon/patient bias in the type of procedure chosen for the patients in these studies. The confounding variables cannot be accounted for in a metaanalysis which is a weakness of this type of study design (metaanalysis) for observational studies, particularly in cases of rare disease. However, the lack of precision in the point estimate for odds ratio for recurrence risk does become more precise with pooling of data from multiple studies.

Metastatic disease is rare, and was in fact only observed in two patients in one study in this meta-analysis [7]. These results lead us to conclude that treatment for these benign aggressive tumors should focus on preserving functionality while attempting to prevent a local recurrence. These may be regarded as conflicting goals, however clinical judgement and experience must be used to decipher those lesions which are likely to be completely treated with intralesional curettage and attempt this procedure when feasible. In fact, one may suggest that intralesional curettage be attempted in all patients who still do have wrist function reasonably undistorted by the underlying pathologic process. One must ensure that the patient is involved in this decision process and understands both the possibility for improved functional outcomes with intralesional treatment, as well as the tradeoff of odds of local recurrence being three times less in the enbloc resection group $v s$ the intralesional curettage group (odds ratio 0.29 ) in our analysis.

An area further for research is to devise a best practice criteria for which lesions may be effectively treated with intralesional curettage without a high probability of recurrence and costing the patient a second surgical procedure. With respect to Campanacci grade, two studies (Cheng et al., and Kang et al.) included only Campanacci grade 3 lesions, and the other four studies reported Campanacci grades but did not report recurrence rates for these grades. Therefore the effect of Campanacci grade cannot be evaluated in this study, and we can only state the available data from included studies.

Most studies suggest that Campanacci 2 lesions can be treated with intralesional curettage whereas wide resection may be considered in Campanacci 3 lesions. However, certain papers reviewed in this meta-analysis report criteria for determining in which Campanacci 3 lesions it would be reasonable to attempt intralesional curettage. Kang et al. [10] postulate that Campanacci 3 lesions should be subdivided by the suffix (p), for those lesions limited to a single site of palmar cortical perforation and bound by the pronator quadratus. Their data suggests that ' $3(\mathrm{p})$ ' lesions can effectively be treated as type 2 lesions (with cortical distortion without cortical disruption). In these lesions, intralesional curettage, cryosurgery and cementation is a treatment possibility with improved functional outcomes, as compared to wide resection, and has an acceptably low rate of recurrence. Other studies similarly support performing wide resection for grade 3 lesions which extend intraarticularly, have greater than $50 \%$ metaphyseal destruction, or have both volar and dorsal cortical perforation [14], and when clinical judgement dictates that curettage and bone grafting would be infeasible [15].

One must be cautious to recognize that intralesional curettage is not without complications. Although en bloc resection and reconstruction may have significant functional implications, a poor outcome from intralesional curettage may necessitate en bloc resection or wrist arthrodesis in the event of recurrence or complication. Sheth et al. [7] cited both skeletal and soft tissue complications from intralesional curettage including carpal collapse, nerve palsy and skin necrosis. Hardware failure and non union were cited as possible complications of the wide resection and reconstruction group. These results further emphasize the importance of selecting the proper therapy on initial presentation.

\section{CONCLUSION}

This meta-analysis is limited by the small number of available studies and the small sample sizes. Our reported odds ratio of 0.29 translates to the odds of local recurrence being approximately three times less in the en-bloc resection group as compared to the intralesional curettage group. This would seem to be a clinically important benefit however should be interpreted with caution due to the limitations of this meta-analysis. Patients undergoing intralesional curettage should be educated regarding the risk for local recurrence and the possibility of en-bloc resection as a salvage procedure. Clinicians must approach this clinical entity on a case by case basis and recognize that it is reasonable to perform intralesional curettage only when the benefit in terms of functional outcomes outweighs the risk of recurrence and morbidity of a second surgical procedure.

\section{CONFLICT OF INTEREST}

The authors confirm that this article content has no conflict of interest.

\section{ACKNOWLEDGEMENTS}

Declared none.

\section{REFERENCES}

[1] Dahlin DC, Cupps RE, Johnson EWJ. Giant-cell tumor: a study of 195 cases. Cancer 1970; 25: 1061-70.

[2] Zhang Q, Zhao H, Maheshwari AD, Cai L, Yu F, Niu, X. Isolated Cardiac Metastasis from a Histologically "Benign" Giant-Cell Tumor of the Distal End of the Femur: A Case Report. J Bone Joint Surg 2010; 92: 2725-31.

[3] Viswanathan S, Jambhekar NA. Metastatic giant cell tumor of bone: are there associated factors and best treatment modalities? Clin Orthop Relat Res 2010; 468: 827-33.

[4] Bertoni F; Present D, Enneking WF. Giant-cell tumor of bone with pulmonary metastases. J Bone Joint Surg Am 1985; 67: 890-900.

[5] Kay RM, Echardt JJ, Seeger LL, Mirra JM, Hak DJ. Pulmonary metastasis of benign giant cell tumor of bone; six histologically confirmed including of one of spontaneous regression. Clin Orthop Relat Res 1994; 302: 219-30.

[6] Vander Griend RA, Funderburk CH. The treatment of giant-cell tumors of the distal part of the radius. J Bone Joint Surg Am 1993; 75: 899-908.

[7] Sheth DS, Healey H, Sobel M, Lane JM, Marcove RC. Giant cell tumor of the distal radius. J Hand Surg 1995; 20A: 432-40. 
[8] Smith RJ, Mankin HJ. Allograft replacement of the distal radius for giant cell tumor. J Hand Surg 1977; 2A: 299-308.

[9] The Newcastle-Ottawa Quality Assessment Scale (NOQAS). Available from: http://www.ohri.ca/programs/clinical_epidemiolog y/oxford.htm

[10] Kang L, Manoso MW, Boland PJ, Healey JH, Athanasian EA. Features of Grade 3 Giant Cell Tumors of the Distal Radius Associated With Succesful Intralesional Treatment. J Hand Surg 2010; 35A: 1850-7.

[11] Campanacci M, Baldini N, Boriani S, Sudanese A. Giant cell tumor of bone. J Bone Joint Surg Am 1987; 69A: 106-14.
[12]

Gitelis S, Mallin BA, Piasecki P. Intralesional excision compared with en bloc resection for giant-cell tumors of bone. J Bone Joint Surg Am 1993; 75: 1648-55.

[13] Liu YP, Li KH, Sun BH. Which treatment is the best for giant cell tumors of the distal radius? A meta-analysis. Clin Orthop Relat Res 2012; 470(10): 2886-94.

[14] Cheng CY, Shih HN, Hsu KY. Treatment of giant cell tumor of the distal radius. Clin Orthop Relat Res 2001; 383: 221-8.

[15] Harness NG, Mankin HJ. Giant-cell tumor of the distal forearm. J Hand Surg Am 2004; 188-93.

[16] Panchwagh Y, Puri A, Agarwal M, Anchan C, Shah M. Giant cell tumor- distal end radius: do we know the answer? Indian J Orthop 2007; 41: 139-45.

(C) Pazionis et al.; Licensee Bentham Open.

This is an open access article licensed under the terms of the Creative Commons Attribution Non-Commercial License (http://creativecommons.org/licenses/by-nc/3.0/) which permits unrestricted, non-commercial use, distribution and reproduction in any medium, provided the work is properly cited. 